\title{
Irreversible electroporation of malignant liver tumors: Effect on laboratory values
}

\author{
MOHAMMED ALNAGGAR ${ }^{1,2}$, AMMAR M. QAID ${ }^{3}$, JIBING CHEN $^{2}$, LIZHI NIU $^{2}$ and KECHENG XU ${ }^{2}$ \\ ${ }^{1}$ Biomedical Translational Research Institute and The First Affiliated Hospital, Jinan University, Guangzhou, \\ Guangdong 510632; ${ }^{2}$ Department of Oncology, Guangzhou Fuda Cancer Hospital, School of Medicine, \\ Jinan University, Guangzhou, Guangdong 510665; ${ }^{3}$ Department of Oncology, Affiliated Union \\ Hospital of Fujian Medical University, Fuzhou, Fujian 350001, P.R. China
}

Received October 11, 2017; Accepted April 25, 2018

DOI: $10.3892 / \mathrm{ol} .2018 .9058$

\begin{abstract}
Liver cancer is often associated with chronic liver diseases. Treatment with percutaneous irreversible electroporation (IRE) may preserve liver function. In the present study, the clinical data of 29 patients with liver tumors between July 2015 and December 2016, all of whom underwent liver IRE at Fuda Cancer Hospital, Guangzhou, China was retrospectively reviewed. All the patients survived the treatment. Of the 29 patients, 7 were positive for hepatitis B, 15 had hepatocellular carcinoma (HCC) and 7 had pancreatic cancer with liver metastases. All patients survived IRE. Despite liver-protective treatment prior to IRE, the mean alanine transaminase (ALT) and aspartate transaminase (AST) levels were significantly elevated 1-2 days after IRE, to 540 and $712 \mathrm{U} / 1$, respectively; however they had returned to the preoperative values by 2 weeks following IRE. Prior to IRE, the mean total bilirubin and direct bilirubin measurement levels were normal; however, 8-10 days after IRE, they had increased to $24 \mathrm{U} / \mathrm{l}$ and $12 \mu \mathrm{mol} / \mathrm{l}$, respectively, and had returned back to the preoperative levels by 2 weeks after IRE. This first group included all patients. The result of the 4 subgroups of cancer patients demonstrated a variation between different measurement days and recovery with patients positive for the hepatitis $\mathrm{B}$ virus taking the longest duration to recover (17 \pm 3 days) meanwhile patients with pancreatic cancer with liver metastases took the shortest time to achieve recovery (10.78 \pm 2 days). The findings of the present study indicate that hepatic injury caused by IRE is transient and self-limiting in patients with liver tumors.
\end{abstract}

Correspondence to: Dr Lizhi Niu, Department of Oncology, Guangzhou Fuda Cancer Hospital, School of Medicine, Jinan University, 2 Tangdexi Road, Guangzhou, Guangdong 510665, P.R. China

E-mail: niuboshi@fudahospital.com

Key words: liver metastases, percutaneous irreversible electroporation, hepatic injury, transaminases, bilirubin

\section{Introduction}

Hepatocellular carcinoma ( $\mathrm{HCC}$ ) is a common tumor worldwide and its incidence rate is increasing (1). Of the affected patients, $80 \%$ have chronic liver diseases such as hepatitis or cirrhosis; the presence of these conditions in conjunction with HCC has a major effect on the prognosis and treatment of this type of cancer (2) Currently, percutaneous ablation therapies, including percutaneous ethanol injection, microwave coagulation and radiofrequency ablation, are available and are important therapeutic modalities for the treatment of HCC (3-8). The hepatitis $\mathrm{B}$ virus (HBV) is responsible for $30 \%$ of all cases of cirrhosis and $>50 \%$ of $\mathrm{HCC}$ cases with an incident rate of 50 million new cases diagnosed annually in endemic countries (9). Irreversible electroporation (IRE) is a newly developed non-thermal ablation procedure where electrical pulses are delivered for a few milliseconds to induce nanoscale defects that increase cell membrane permeability, the procedure induces apoptosis without harming the extracellular matrix; therefore, the structural components of the tissues are preserved $(10,11)$. IRE is a novel, non-thermal form of tumor ablation that is not affected by heat sink and may result in less collateral damage based on its mechanism of action (12). IRE relies on short pulses of high-frequency energy to induce pores in the lipid bilayer of cells, leading to cell death via apoptosis. In the present study, hepatic injury in 29 patients following a single IRE session was retrospectively assessed. The serum transaminases and bilirubin values were compared between patients who were positive and those who were negative for hepatitis B and for patients with HCC and pancreas cancer with liver Metatases, to study the effects of IRE on liver function and its recovery.

\section{Materials and methods}

Ethics. The present study was approved by the Regional Ethics Committee of Guangzhou Fuda Cancer Hospital (Guangzhou China). Written informed consent was obtained from all patients, and the study protocols were in accordance with the tenets of the Declaration of Helsinki.

Patient selection. Diagnosis of unresectable HCC and liver metastasis was confirmed by pathological studies. 
The diagnoses were confirmed blindly by two pathologists at pathology department affiliated with Jinan University (Guangzhou, China) and imaging analyses and measurement of the tumor marker $\alpha$-fetoprotein. Patients who met the following criteria were considered to be eligible for the study: The presence of one or two significant tumors in the liver, Karnofsky performance status (13) (KPS) score $\geq 70$, white blood cell count $\geq 3 \times 10^{9} / 1$, neutrophil count $\geq 2 \times 10^{9} / 1$; hemoglobin $\geq 90 \mathrm{~g} / \mathrm{l}$, platelet count $\geq 100 \times 10^{9} / 1$, prothrombin time (international normalized ratio, INR) $\geq 1.5$. Furthermore, the present study excluded patients who had severe coronary heart disease, myelosuppression, respiratory disease, acute/chronic infection, or level 3 hypertension, and had adequate hepatic function [total bilirubin (T.BIL) $<75 \mu \mathrm{mol} / 1$, direct bilirubin (D.BIL) $<39 \mu \mathrm{mol} / 1$, and a Child-Pugh score (14) of A or B] and renal function (serum creatinine $<130 \mu \mathrm{m}$ and serum urea $<10 \mathrm{~mm}$ ). Between July 2015 and December 2016, 29 patients (20 males; age range, 32-75 years; mean age, 55 years; and 9 females; age range, $32-70$ years; mean age, 53 years) were eligible and enrolled from Fuda cancer hospital, Affiliated with Jinan University (Guangzhou, China).

Irreversible electroporation procedure. All patients underwent gastric decompression and endotracheal intubation. General anesthesia was induced intravenous and inhalation anesthesia by trachea intubation, drugs delivered via vein and trachea by intravenous infusion, including midazolam $(0.5 \mathrm{mg})$ and penehyclidine hydrochloride $(0.5 \mathrm{mg})$. For general anesthesia induction: Etomidate $(0.3 \mathrm{mg} / \mathrm{kg})$, benzenesulfonic acid cisatracurium $(0.2 \mathrm{mg} / \mathrm{kg})$ and remifentanil $(150 \mu \mathrm{g})$ were used, and for maintenance: Intravenous pumping of diprivan $(50-150 \mathrm{mg} / \mathrm{h}) ;$ remifentanil $(320-720 \mu \mathrm{g} / \mathrm{h})$ and inhalation of sevoflurane (0.6-2\%) benzenesulfonic acid cisatracurium $(6 \mu \mathrm{g} / \mathrm{kg})$. Computed tomography (CT) was undertaken and the target region was demarcated. Ultrasound-guided insertion of 2-3 electrodes was performed. Following anesthesia an additional CT image was taken to confirm correct placement of the electrodes. IRE was synchronized to deliver electrical pulses coordinated with cardiac rhythm to prevent cardiac dysrhythmia. Typically, the distance between electrodes was $1.5-2 \mathrm{~cm}$. The voltage was set at 1,500-3,000 kV. Between $70-90$ pulses were delivered in 7-9 sets of 10 pulses. Additional pullbacks were performed if the target region was $>2 \mathrm{~cm}$ in diameter. The baseline and highest heart rate during IRE were recorded by electrocardiography. Arrhythmia of any form was documented. Invasive blood pressure measurement via the femoral artery was applied for precise systolic blood pressure (SBP) monitoring. If SBP was $>40 \mathrm{mmHg}$ during ablation or $>190 \mathrm{mmHg}$ at any time, electric pulses were suspended for 2-3 min. If no obvious decrease in SBP was observed after 2-3 min, 2-5 mg of phentolamine was administered intravenously to lower blood pressure and prevent hypersensivity.

Assessment of hepatic functional reserve. Hepatic function was assessed with an automatic biochemical analyzer (7100; Hitachi Ltd., Tokyo, Japan). The alanine transaminase (ALT) and aspartate transaminase (AST) levels were measured with the velocity method using a specialized reagent kit (AST kit: Aspartate Aminotransferase kit [Aspartate Substrate Method, cat. no. 1740-2013. ALT kit: Alanine aminotransferase kit.
(Alanine low content method) no. 170781], and the T.BIL and D.BIL levels were measured using the vanadate method with a commercially available kit [TBIL: Total Bilirubin kit (Vanadate Oxidation Method) no. 1737-2013. D.Bil: Direct Bilirubin kit (Vanadate Oxidation Method) no. 4523-40-201] (BioSino Bio-Technology and Science Inc., Beijing, China). Blood samples were obtained in the morning, after overnight fasting. The tests were performed every 1-3 days, until the patients were discharged (on day 20). The normal ranges for the measured parameters were as follows: ALT, 5-35 U/1; AST, 8-40 U/l; T.BIL, 0-25.5 $\mu \mathrm{mol} / \mathrm{l}$; and D.BIL, 0-13 $\mu \mathrm{mol} / \mathrm{l}$. Values that were above the upper limit of the normal range were considered to indicate abnormal hepatic function.

Statistical analysis. The revised version of the Response Evaluation Criteria in Solid Tumors (version 1.1) (15) was used to determine the response of the hepatic tumors to the treatment. Statistical tests were performed with commonly used methods, and a nonparametric, one-way AVONA followed by Bonferroni's multiple comparison post-hoc test was used for comparisons between days. Test results are expressed as the mean \pm standard error and $\mathrm{P}<0.05$ was considered to indicate a statistically significant difference. All analyses were conducted using GraphPad software version 5.0 (GraphPad, Software, Inc., La Jolla, CA, USA).

\section{Results}

Clinical data. Prior to hepatic IRE, detailed data of the patients were extracted and are presented in Table I. Of the 29 patients, 22 (75.9\%) had HCC, 7 (24.1\%) had a history of hepatitis, 7 (24.1\%) had pancreatic cancer with liver metastases, 10 (34.5\%) had undergone initial surgical treatment and $22(75.86 \%)$ had undergone systemic chemotherapy at different centers.

Perioperative outcomes. All the hepatic lesions were treated with IRE, which was performed successfully in all patients. Severe complications (such as rupture or hepatic failure, myoglobinuria and acute renal failure) did not occur following IRE. There were several mild adverse effects [7 patients developed fever $\left(38.2-39.8^{\circ} \mathrm{C}\right), 15$ patients had pain for 3-7 days, 1 patient had infection, 2 patients had abdominal ascites, and 6 patients developed variable degree of nausea and vomiting]; however the patients recovered without symptomatic management.

Changes in hepatic functional reserve after irreversible electroporation. Of the 29 patients, 7 were positive for the hepatitis B virus, 15 had HCC), and 7 had pancreatic cancer with liver metastases. A total of 29 IRE procedures were performed. The transaminase levels were abnormal until the 4th session of ablation, and the bilirubin level was abnormal until the 2nd session of ablation (this was the case for all patients). The serum transaminase levels increased rapidly and reached a peak on day 1 following IRE, after which they gradually decreased. The ALT and AST levels demonstrated a significant decrease from day 5 and 7 , respectively $(\mathrm{P}<0.001$; Fig. 1A and B). No significant change in the serum bilirubin level was observed until 20 days after IRE (Fig. 1C and D). 
Table I. Detailed data on patients prior to hepatic irreversible electroporation.

\begin{tabular}{|c|c|}
\hline Characteristic & $\mathrm{n}(\%)$ \\
\hline \multicolumn{2}{|l|}{ Sex } \\
\hline Female & $9(31.03)$ \\
\hline Male & $20(69.97)$ \\
\hline $\mathrm{HCC}$ & $22(75.9)$ \\
\hline Sex (male/female) & $15 / 7$ \\
\hline Median age (years) & 52 \\
\hline \multicolumn{2}{|l|}{ AJCC stage $(2010)(37,38)$} \\
\hline IIIA & $2(9.09)$ \\
\hline IIIB & $1(4.55)$ \\
\hline IIIC & $2(9.09)$ \\
\hline IVA & $17(77.27)$ \\
\hline Hepatitis B positive & $7(24.1)$ \\
\hline \multicolumn{2}{|l|}{ Child-Pugh stage (15) } \\
\hline A & $14(63.64)$ \\
\hline $\mathrm{B}$ & $8(36.36)$ \\
\hline \multicolumn{2}{|l|}{ Liver function } \\
\hline ALT, U/1 & $27 \pm 11$ \\
\hline $\mathrm{AST}, \mathrm{U} / 1$ & $44 \pm 43$ \\
\hline T.BIL, $\mu \mathrm{mol} / 1$ & $18 \pm 7$ \\
\hline D.BIL, $\mu \mathrm{mol} / \mathrm{l}$ & $7 \pm 4$ \\
\hline \multicolumn{2}{|l|}{ Tumor type } \\
\hline Single massive & $17(77.27)$ \\
\hline Multinodular & $5(22.73)$ \\
\hline \multicolumn{2}{|l|}{ Ascites } \\
\hline Yes & $14(63.64)$ \\
\hline No & $8(36.36)$ \\
\hline Pancreatic cancer with liver metastasis & $7(24.1)$ \\
\hline Sex (male/female) & $5 / 2$ \\
\hline Median age (years) & 53 \\
\hline \multicolumn{2}{|l|}{ AJCC stage $(24,38)$} \\
\hline III & 0 \\
\hline IV & 7 \\
\hline \multicolumn{2}{|l|}{ Liver function } \\
\hline ALT, U/1 & $35 \pm 11$ \\
\hline $\mathrm{AST}, \mathrm{U} / 1$ & $49 \pm 23$ \\
\hline T.BIL,$\mu \mathrm{mol} / 1$ & $14 \pm 4$ \\
\hline D.BIL, $\mu \mathrm{mol} / \mathrm{l}$ & $7 \pm 4$ \\
\hline
\end{tabular}

AJCC, American Joint Committee on Cancer staging system; HCC, hepatocellular carcinoma; ALT, alanine aminotransferase; AST aspartate aminotransferase; T.BIL, total bilirubin; D.BIL, direct bilirubin.

Changes in hepatic functional reserve in seven patients with hepatitis $B$. Each of the 7 patients who were positive for hepatitis B underwent IRE. Prior to the 2nd and 3rd ablation sessions, the transaminase and bilirubin levels were abnormal (data not shown). Additionally, 1 day after IRE was performed, the serum transaminase levels increased rapidly until they reached a peak $(\mathrm{P}<0.001)$, after which they gradually decreased. The
ALT and AST levels showed a significant decrease from day 5 $(\mathrm{P}<0.01)$ and day 3, respectively $(\mathrm{P}<0.001$; Fig. 2A and $\mathrm{B})$. There was no obvious variation in the serum bilirubin level until 20 days following IRE (Fig. 2C and D).

Changes in hepatic functional reserve in 22 patients without hepatitis $B$. All the 22 patients who were negative for hepatitis B underwent liver IRE. None of the patients had normal transaminase levels; in addition, the bilirubin level was abnormal before the 8th ablation session. The serum ALT and AST level increased within 2 days after IRE $(\mathrm{P}<0.01$; Fig. $3 \mathrm{~A}$ and B) and then decreased gradually, a markedly significant decrease was noted after 8-10 days. No significant change in the serum bilirubin level was observed (Fig. 3C and D).

Variations in hepatic functional reserve patients with HCC. In the 15 patients with HCC who underwent IRE, the serum transaminase levels increased rapidly till they reached a peak the day after IRE, following which they decreased gradually. The serum ALT and AST levels showed a significant decrease from day $0,1,3$ and 5, respectively ( $\mathrm{P}<0.01$; Fig. $4 \mathrm{~A}$ and $\mathrm{B}$ ). However, no significant change was observed in the serum bilirubin levels until 20 days following IRE (Fig. 4C and D).

Changes in hepatic functional reserve in patients with pancreatic cancer with liver metastases. The 7 patients with pancreatic cancer with liver metastases also underwent liver IRE. The serum ALT and AST value increased within 2 days following IRE $(\mathrm{P}<0.01)$ and then fell gradually, with a significant decrease observed after day 10 (Fig. 5A and B; $\mathrm{P}<0.05)$. The serum bilirubin levels increased gradually until they reached a peak at 10-12 days after IRE (Fig. 5C and D; $\mathrm{P}<0.05$ ), following which it decreased gradually.

Changes in hepatic functional reserve in the 4 patient subgroups. Each result of the 4 subgroups of patients with cancer demonstrated a variation between different measurement days and recovery with patients positive for the hepatitis $\mathrm{B}$ virus taking the longest duration to recover (recovery was considered to be when liver functions returned to normal) i.e., $17 \pm 3$ days, meanwhile, the patients negative for the hepatitis $\mathrm{B}$ viruses and patients with $\mathrm{HCC}$ whose recovery is observed in $15 \pm 2.9$ and $12.62 \pm 4.3$ days respectively, by contrast patients with pancreatic cancer with liver metastases took the shortest time to achieve recovery $10.78 \pm 2$ days. The result was statistically significant between pancreatic cancer with liver metastases and the other groups $\left(\mathrm{HCC}, \mathrm{HBV}^{+}\right.$and $\mathrm{HBV}^{-}$; $\mathrm{P}<0.05$; Fig. 6). Overall patients suffering from primary hepatic diseases took longer duration to recover when compared with patients with metastatic disease.

\section{Discussion}

IRE is a promising procedure for unresectable hepatocellular carcinoma (16). The complications of IRE when used to treat malignant hepatic tumors (such as fever, local pain, abdominal distension, ascites, nausea and vomiting) are acceptable and there is evidence to indicate that the therapy may improve survival. Thus, it is an effective liver tumor ablative therapy that results in only mild and transient adverse effects (17-20). 

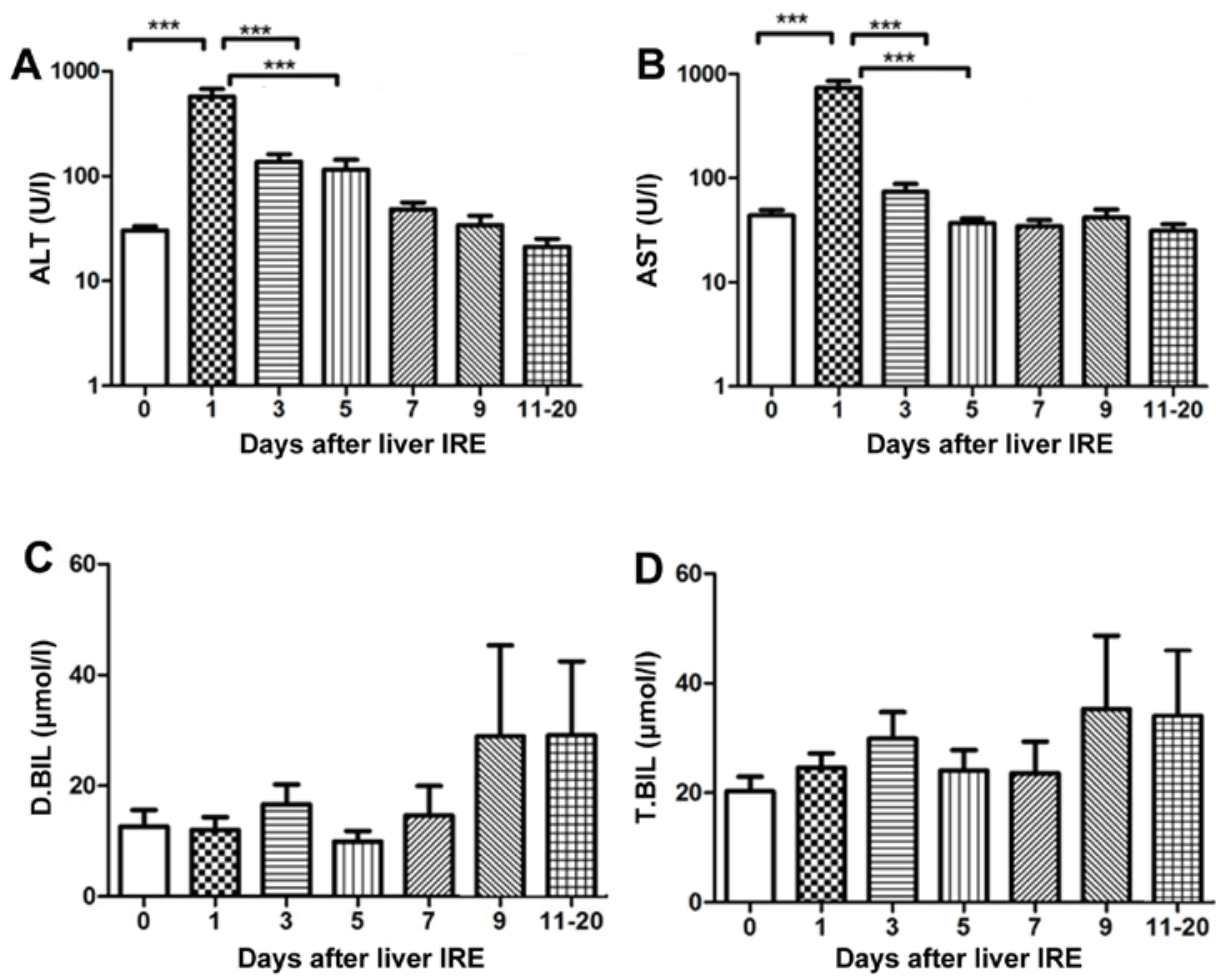

Figure 1. Variations in hepatic functional reserve after irreversible electroporation in the 29 patients of the present study. Bonferroni's multiple comparison was used for comparison of values between the stated time points. The number of results varied as follows: $22,18,18,16,10,15,8,9,6,8$ and 4: 22 results were obtained on days $0,1-2,3-4,5-6,7-8,9-10$ and 11-20, respectively. Days on which $<3$ test results were obtained were merged with adjacent days. The markers of hepatic functional reserve were (A) alanine transaminase, (B) aspartate transaminase, (C) direct bilirubin and (D) total bilirubin. ${ }^{* * *} \mathrm{P}<0.001 \mathrm{vs}$. IRE, irreversible electroporation; ALT, alanine transaminase; ALT, aspartate transaminase; D.BIL, direct bilirubin; T.BIL, total bilirubin.
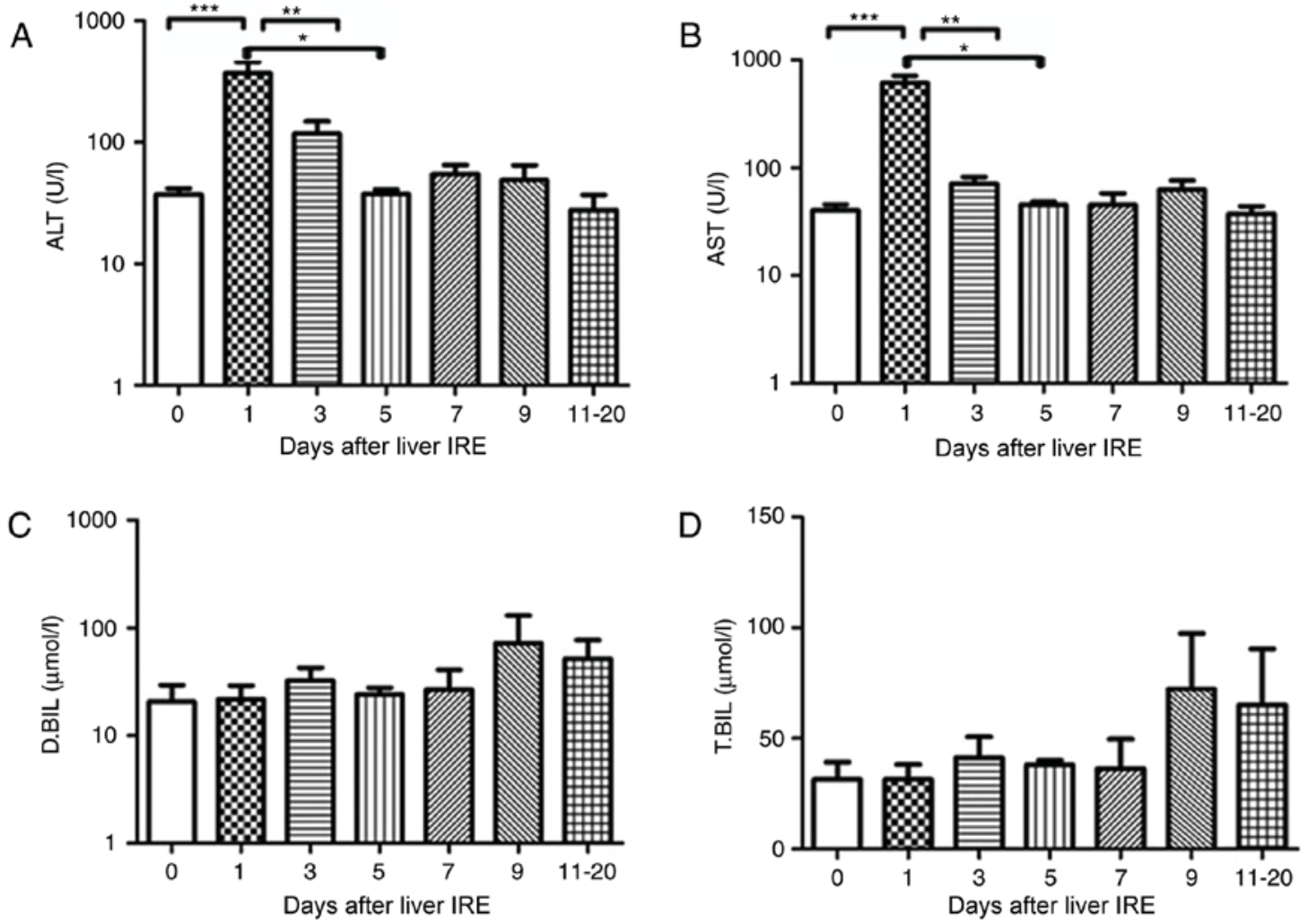

Figure 2. Variations in hepatic functional reserve in seven patients with hepatitis B. Bonferroni's multiple comparison post-hoc test was used to analyze differences in values between time points. The number of results obtained varied as follows: 18, 8, 6, 4, 4, 5 and 6: 6 results were obtained on days $0,1-2$, 3-4, 5-6, 7-8, 9-10 and 11-20, respectively. Days on which $<3$ test results were obtained were merged with adjacent days. The following markers of hepatic functional reserve were used: (A) alanine transaminase, (B) aspartate transaminase, (C) direct bilirubin and (D) total bilirubin. ${ }^{* * *} \mathrm{P}<0.001,{ }^{* *} \mathrm{P}<0.01$ and ${ }^{*} \mathrm{P}<0.05$ vs. Day $0,1,3$ or 5 . IRE, irreversible electroporation; ALT, aspartate transaminase; D.BIL, direct bilirubin; T.BIL, total bilirubin. 

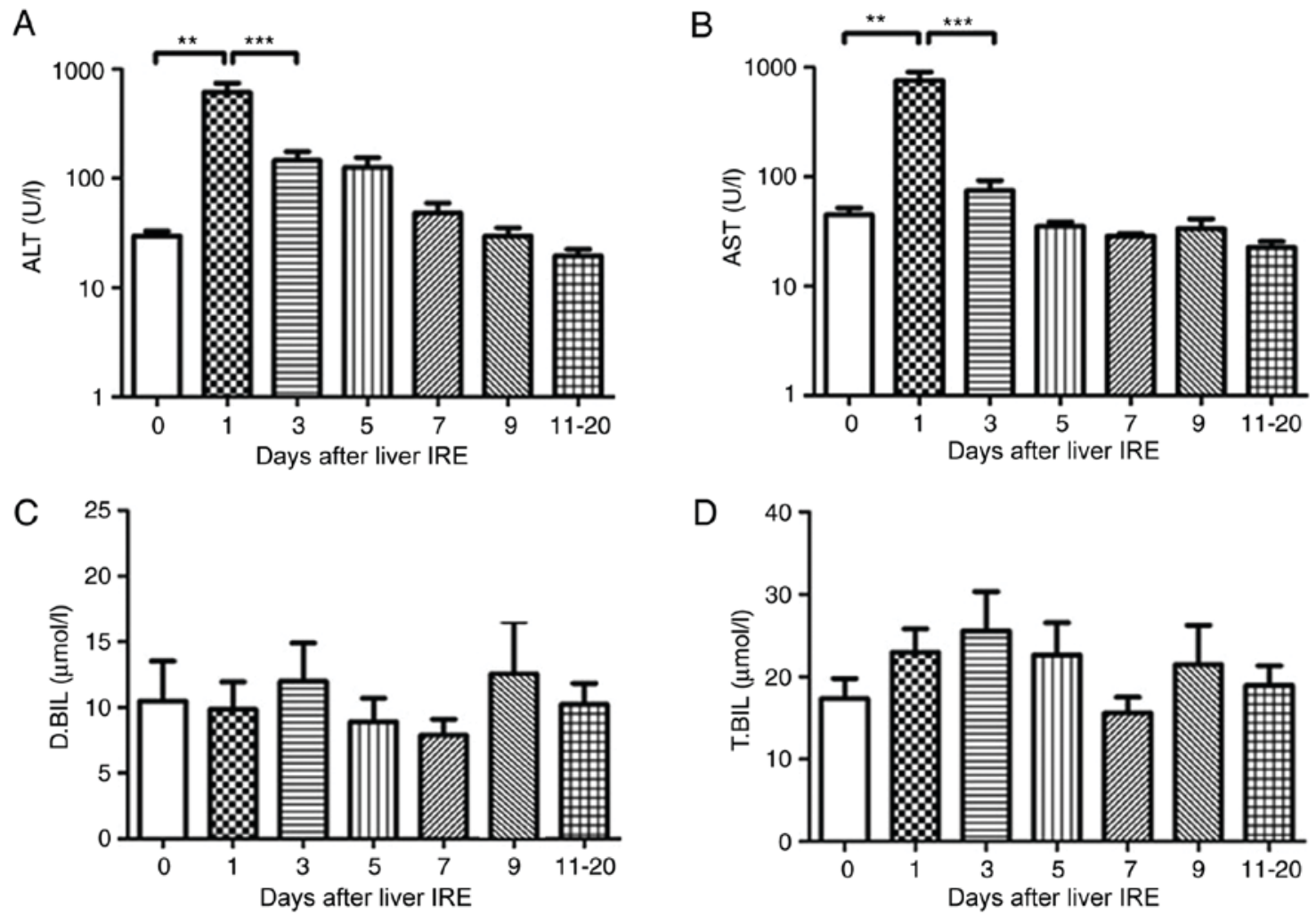

Figure 3. Variations in hepatic functional reserve in 22 patients without hepatitis B. Bonferroni's multiple comparison was used to compare values across time points. The number of results varied between days: 48,16,12, 10, 6, 11, 10 and 12 : 18 results were obtained on days $0,1,2,3,4,5,6-7,8-10$ and 11-20, respectively. Days on which $<3$ test results were obtained were merged with adjacent days. The hepatic functional reserve markers were (A) alanine transaminase, (B) aspartate transaminase, (C) direct bilirubin and (D) total bilirubin. ${ }^{* *} \mathrm{P}<0.01$ and ${ }^{* * * *} \mathrm{P}<0.001$ vs. Day 0,1 or 3 . IRE, irreversible electroporation; ALT, aspartate transaminase; D.BIL, direct bilirubin; T.BIL, total bilirubin.
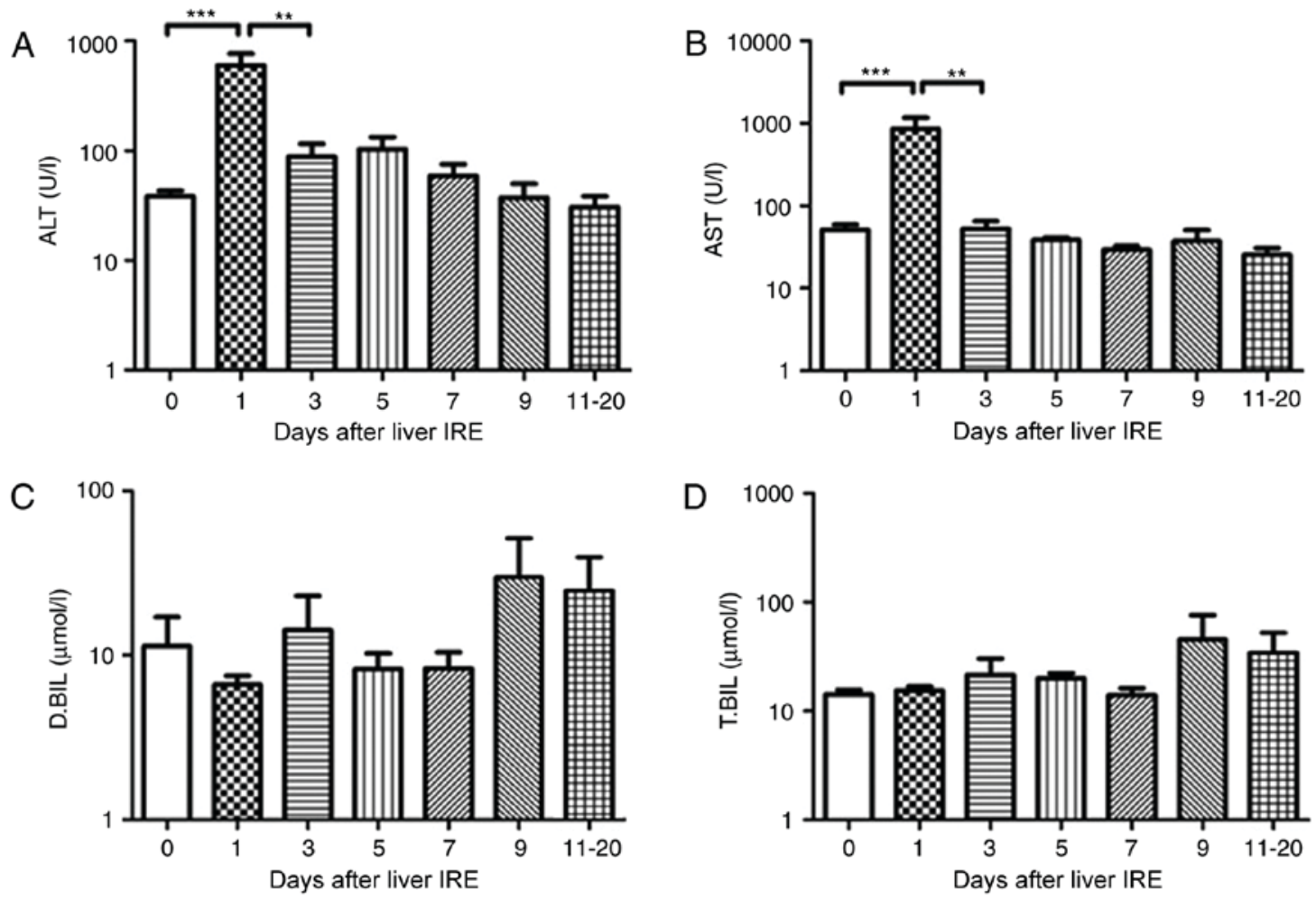

Figure 4. Variations in hepatic functional reserve after irreversible electroporation in 15 patients with hepatocellular carcinoma. Bonferroni's multiple comparison was used to compare values between different time points. The number of results obtained varied across time points as follows: 20, 16 16, 16, 15, 8, 9, 6 and 4: 22 results were obtained on Days 0, 1-2, 3-4, 5-6, 7-8, 9-10 and 11-20, respectively. Days on which <3 test results were obtained were merged with adjacent days. The hepatic functional reserve markers used were (A) alanine transaminase, (B) aspartate transaminase, (C) direct bilirubin and (D) total bilirubin. ${ }^{* * * *} \mathrm{P}<0.001$ and ${ }^{* *} \mathrm{P}<0.01$ vs. Day 0,1 or 3 IRE, irreversible electroporation; ALT, aspartate transaminase; D.BIL, direct bilirubin; T.BIL, total bilirubin. 

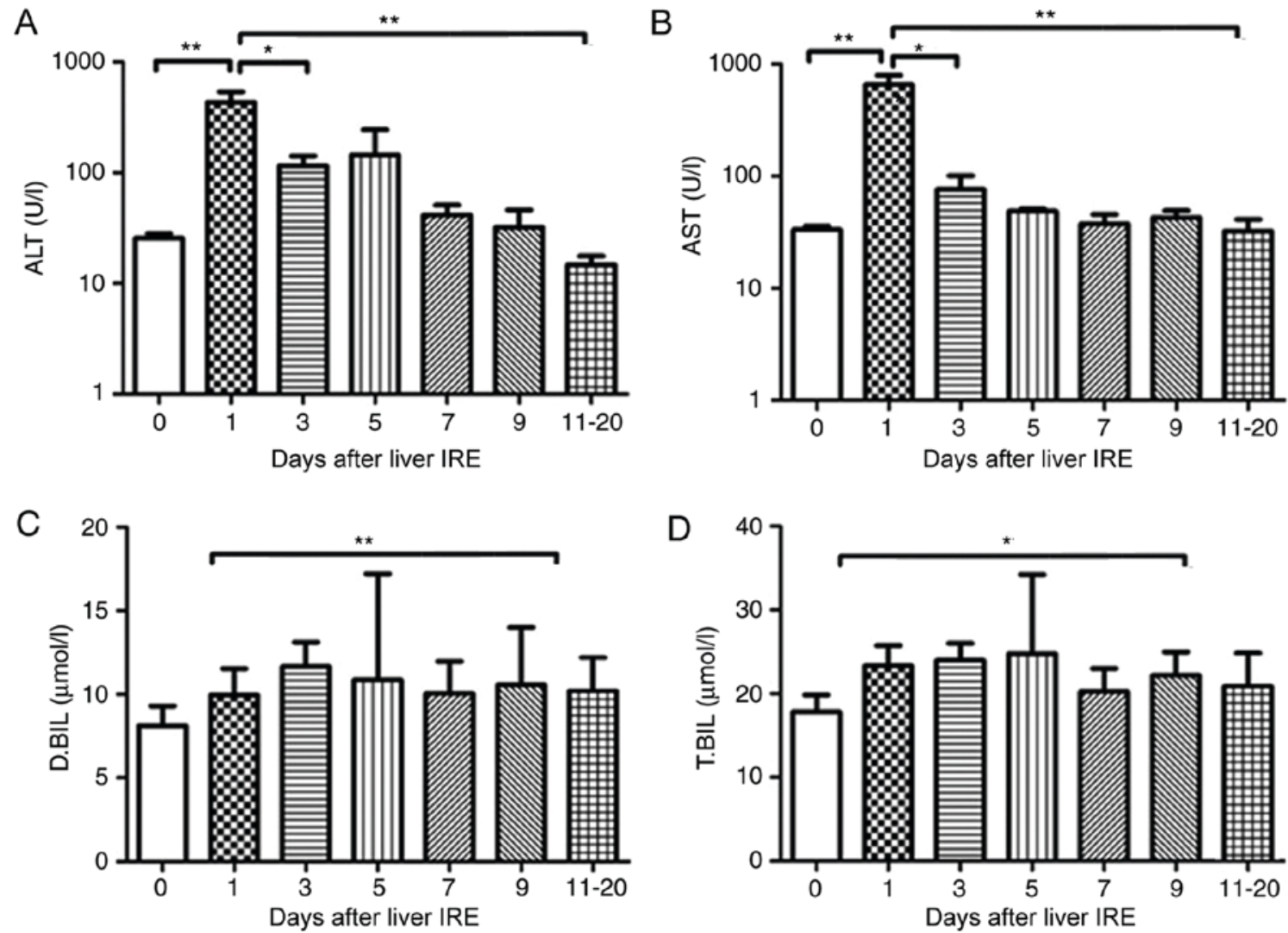

Figure 5. Variations in hepatic functional reserve in 7 patients with pancreatic cancer with liver metastases. Bonferroni's multiple comparison was used to compare values between different time points. The number of test results varied between different measurement days as follows: 18, 8, 6, 4, 4, 5 and 6: 6 results were obtained on days $0,1-2,3,4,5,6,7-9$ and 10-20, respectively. Days on which $<3$ test results were obtained were merged with adjacent days. The following markers of hepatic functional reserve were used: (A) alanine transaminase, (B) aspartate transaminase, (C) direct bilirubin and (D) total bilirubin. "P<0.05, ${ }^{* *} \mathrm{P}<0.01$ vs. Day $0,1,3$ or 10. IRE, irreversible electroporation; ALT, aspartate transaminase; D.BIL, direct bilirubin; T.BIL, total bilirubin.

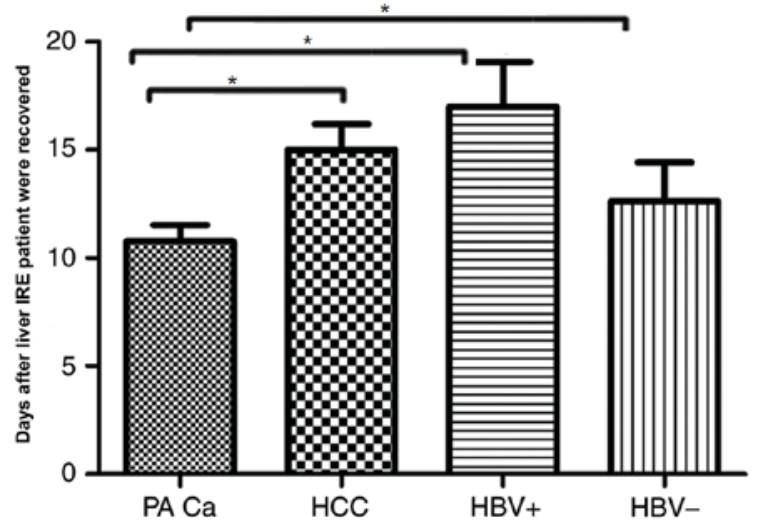

Figure 6. Variations in hepatic functional reserve in the 4 patient subgroups. The patients with pancreatic cancer with liver metastases was the first group to recover to normal liver function, while the HBV+ group was the last. "P $<0.05$. IRE, irreversible electroporation; PA Ca; HCC, hepatocellular carcinoma; $\mathrm{HBV}+$, Hepatitis B positive; HBV-, Hepatitis B negative.

IRE is a novel ablation technique wherein electroporation or electro-permeabilization is used to generate electric pulses that create nanoscale defects in the cell membrane and alter its permeability (21). Ablative treatment has been identified to be safe and effective in patients with primary and secondary liver cancer, and those who are not suitable for surgery (22). In the present study, one session of IRE was performed for primary liver tumors and secondary metastases to avoid serious adverse effects. Follow-up liver function tests and routine blood tests revealed immediate liver function damage followed by recovery, consistent with the findings of a study by Chen et al (23). IRE causes significant abnormalities in liver function; however, in the majority of patients these are self-limiting, do not preclude treatment and resemble the changes observed following radiofrequency ablation or cryoablation of the liver (24).

IRE for the treatment of liver cancer is associated with hepatocyte destruction and the subsequent release of transaminases and bilirubin, which are considered as important markers of hepatic functional reserve (25). Similarly, a recent study has reported marked elevations in AST, ALT, T.BIL, and D.BIL (26), which are usually transient and normalize within a few days. It was identified that 1 day after ablation, the ALT level was 3.3-fold higher (mean peak level, $241 \mathrm{U} / \mathrm{l}$ ) and the AST level was 5-fold higher (mean peak level, $427 \mathrm{U} / 1$ ); however, they had almost reverted to the preoperative levels within 5 days following the procedure (26). A similar study $(22,27)$ identified 15 bile duct injuries (narrowing, $\mathrm{n}=8$; dilation, $\mathrm{n}=7$ ) in subacute follow-up magnetic resonance images examinations of 3 patients demonstrated transient abnormalities of laboratory values of bilirubin, $1.6-5.2 \mathrm{mg} / \mathrm{dl}$. Short-term laboratory values were abnormal in 1 patient (increased alkaline phosphatase of $533 \mathrm{U} / 1$ vs. baseline) as a result of local tumor recurrence. 
Recently, Silk et al (28) reported where IRE was used to treat 22 hepatic metastases in 11 patients; the laboratory values were abnormal after 4 treatment sessions in 3 patients (bilirubin, 2.6-17.6 mg/dl; alkaline phosphatase, 130-1,035 U/l). These abnormal values were transient after 2 sessions, with 2 patients exhibiting prolonged elevation of these abnormal values and 1 requiring stent placement. In the former 2 patients, these adverse effects appeared to be secondary to tumor progression rather than bile duct injury (28). Conversely, the present study did not identify a prolonged significant increase in serum bilirubin. The participants were classified into the hepatitis B virus-positive and hepatitis B-negative groups, and an increase in T.BIL by $>3$ times [ $74 \mu \mathrm{mol} / 1$ following IRE vs. $23 \mu \mathrm{mol} / 1$ prior to IRE (3.2 times greater)] and a similar increase in D.BIL [ $45 \mu \mathrm{mol} / 1$ after IRE vs. $12 \mu \mathrm{mol} / 1$ prior to IRE (3.8 times greater)] were only observed in the hepatitis B-positive group at 3-5 days following treatment. The increase in AST and ALT levels observed in the present study was consistent with previous studies; however the range of increase varied between studies (18,29-31). The minimal increase that was observed in the present study may be explained by the time points of the measurements. Overall, the results indicate the importance of ALT and AST as markers of post-IRE liver function.

In patients with hepatitis $\mathrm{B}$, serum bilirubin increased $>3$ times compared with the preoperative level 7-9 days following IRE. The reason for this increase remains unclear and warrants further research. In the present study, all the patients tolerated the treatment and there were no records of severe complications following IRE. Compared with radiofrequency ablation, IRE resulted in faster liver regeneration, which demonstrated the safety and efficacy of the technique (32). A recent prospective study of IRE treatment of 44 patients with HCC, colorectal, and other lesions reported the following local, recurrence-free survival rates: $97.4 \%$ at 3 months, $94.6 \%$ at 6 months and $59.5 \%$ at 12 months, the recurrence rates tended to be higher in lesions with diameter $>4 \mathrm{~cm}$ (32). In a smaller cohort of 28 patients treated with IRE, portal vein thrombosis was reported in only 1 case in which the tumor was located within $1 \mathrm{~cm}$ of a major portal pedicle (33). Based on the findings, the authors of the study concluded that IRE was safe even for the treatment of liver lesions present near major vascular structures, and their findings are consistent with the reported theoretical benefits of non-thermal ablation (34-36). Cannon et al (32) reported that in a cohort of 44 patients, who underwent IRE for primary and secondary liver cancer, the incidence of complications was low. Only 5 patients were observed to have developed adverse events, but the complications were resolved within 30 days of treatment (32). Thus, of previously published studies, many have demonstrated that IRE is a safe and efficient therapeutic method for the treatment of patients with pancreatic and colorectal liver metastases (36).

The limitations of the present study are as follows: No biochemical, hematologic or immunologic factors other than ALT, AST and serum bilirubin were investigated. Although the long-term results are as yet unknown, the future of IRE is promising; however, significant concerns remain regarding its safety over an extended period of time.

To conclude, IRE for primary and metastatic liver cancer may be considered safe in terms of its short-term effects on liver function; however, further study is required to confirm the findings of the present study.

\section{Acknowledgements}

Not applicable.

\section{Funding}

The present study was supported by Scientific and Technological Plan, Guangdong province, China (grant no. 2016A020216018) and International Foundation for Sciences of Guangzhou Fuda Cancer Hospital (grant no. Y2016-ZD-008).

\section{Availability of data and materials}

The datasets used and/or analyzed during the current study are available from the corresponding author on reasonable request.

\section{Authors' contributions}

LN, MA and AMQ were responsible for study conception and design. MA and AMQ contributed to the acquisition and interpretation of data; JC drafted the work and revised the manuscript. JC and KX acquired and interpreted the data. All the authors approved the article for publication and $\mathrm{KX}$ agreed to be accountable for all the aspects of the work including its integrity.

\section{Ethics approval and consent to participate}

The present study was approved by the Regional Ethics Committee of Guangzhou Fuda Cancer Hospital, China. Written informed consent was obtained from all the patients, and the study protocols were in accordance with the tenets of the Declaration of Helsinki.

\section{Patient consent for publication}

Participants gave informed consent for data sharing.

\section{Competing interests}

The authors declare that they have no competing interests.

\section{References}

1. Ali AM, Lizhi N, Jialiang L, Fei Y, Yuan W, Jianying Z, Jin Y, Jibing $\mathrm{C}$, Feng $\mathrm{M}$ and Kecheng $\mathrm{X}$ : Cryoprotective therapy for hepatocellular carcinoma: Study of 51 patients with a single lesion. Cryobiology 69: 61-67, 2014.

2. Alnaggar M, Niu L, Li J, Yao F, Wang Y, Zeng J, Ye J, Chen J, $\mathrm{Mu} \mathrm{F}$ and $\mathrm{Xu} \mathrm{K}$ : Cryoprotective therapy for huge hepatocellular carcinoma: A study of 14 patients with a single lesion. Cryobiology 69: 457-461, 2014.

3. Pearson AS, Izzo F, Fleming RY, Ellis LM, Delrio P, Roh MS, Granchi J and Curley SA: Intraoperative radiofrequency ablation or cryoablation for hepatic malignancies. Am J Surg 178: 592-599, 1999.

4. Chung CD, Lau LL, Ko KL, Wong AC, Wong S, Chan AC, Poon RT, Lo CM and Fan ST: Laparoscopic liver resection for hepatocellular carcinoma. Asian J Surg 33: 168-172, 2010.

5. Ravikumar TS, Kane R, Cady B, Jenkins R, Clouse $M$ and Steele G Jr: A 5-year study of cryosurgery in the treatment of liver tumors. Arch Surg 126: 1520-1524, 1991. 
6. Sarantou T, Bilchik A and Ramming KP: Complications of hepatic cryosurgery. Semin Surg Oncol 14: 156-162, 1998.

7. Seifert JK and Morris DL: World survey on the complications of hepatic and prostate cryotherapy. World J Surg 23: 109-114, 1999.

8. Shafir M, Shapiro R, Sung M, Warner R, Sicular A and Klipfel A: Cryoablation of unresectable malignant liver tumors. Am J Surg 171: 27-31, 1996.

9. Schweitzer A, Horn J, Mikolajczyk RT, Krause G and Ott JJ: Estimations of worldwide prevalence of chronic hepatitis B virus infection: A systematic review of data published between 1965 and 2013. Lancet 386: 1546-1555, 2015.

10. Al-Sakere B, André F, Bernat C, Connault E, Opolon P, Davalos RV, Rubinsky B and Mir LM: Tumor ablation with irreversible electroporation. PloS One 2: e1135, 2007.

11. Edd JF, Horowitz L, Davalos RV, Mir LM and Rubinsky B: In vivo results of a new focal tissue ablation technique: Irreversible electroporation. IEEE Trans Biomed Eng 53: 1409-1415, 2006.

12. Charpentier KP, Wolf F, Noble L, Winn B, Resnick M and Dupuy DE: Irreversible electroporation of the liver and liver hilum in swine. HPB (Oxford) 13: 168-173, 2011.

13. Péus D, Newcomb $\mathrm{N}$ and Hofer S: Appraisal of the karnofsky performance status and proposal of a simple algorithmic system for its evaluation. BMC Med Inform Decis Mak 13: 72, 2013

14. Cholongitas E, Papatheodoridis GV, Vangeli M, Terreni N, Patch D and Burroughs AK: Systematic review: The model for end-stage liver disease-should it replace Child-Pugh's classification for assessing prognosis in cirrhosis? Aliment Pharmacol Ther 22: 1079-1089, 2005.

15. Xu HM, Chen Y, Xu J and Zhou Q: Drug-induced liver injury in hospitalized patients with notably elevated alanine aminotransferase. World J Gastroenterol 18: 5972-5978, 2012.

16. Kos B, Voigt P, Miklavcic D and Moche M: Careful treatment planning enables safe ablation of liver tumors adjacent to major blood vessels by percutaneous irreversible electroporation (IRE). Radiol Oncol 49: 234-241, 2015.

17. Dollinger $\mathrm{M}$, Muller-Wille $\mathrm{R}$, Zeman F, Haimerl M, Niessen C, Beyer LP, Lang SA, Teufel A, Stroszczynski C and Wiggermann P: Irreversible electroporation of malignant hepatic tumors-alterations in venous structures at subacute follow-up and evolution at mid-term follow-up. PloS One 10: e0135773, 2015.

18. Dollinger M, Zeman F, Niessen C, Lang SA, Beyer LP, Müller M, Stroszczynski C and Wiggermann P: Bile duct injury after irreversible electroporation of hepatic malignancies: Evaluation of MR imaging findings and laboratory values. J Vasc Interv Radiol 27: 96-103, 2016.

19. Dollinger M, Beyer LP, Haimerl M, Niessen C, Jung EM, Zeman F, Stroszczynski C and Wiggermann P: Adverse effects of irreversible electroporation of malignant liver tumors under CT fluoroscopic guidance: A single-center experience. Diagn Interv Radiol 21: 471-475, 2015.

20. Scheffer HJ, Nielsen K, de Jong MC, van Tilborg AA, Vieveen JM, Bouwman AR, Meijer S, van Kuijk C, van den Tol PM and Meijerink MR: Irreversible electroporation for nonthermal tumor ablation in the clinical setting: A systematic review of safety and efficacy. J Vasc Interv Radiol 25: 997-1011; quiz 1011, 2014.

21. Cohen EI, Field D, Lynskey GE and Kim AY: Technology of irreversible electroporation and review of its clinical data on liver cancers. Expert Rev Med Devices 15: 99-106, 2018.

22. Ryan MJ, Willatt J, Majdalany BS, Kielar AZ, Chong S, Ruma JA and Pandya A: Ablation techniques for primary and metastatic liver tumors. World J Hepatol 8: 191-199, 2016.

23. Chen X, Ren Z, Zhu T, Zhang X, Peng Z, Xie H, Zhou L, Yin S, Sun J and Zheng S: Electric ablation with irreversible electroporation (IRE) in vital hepatic structures and follow-up investigation. Sci Rep 5: 16233, 2015.

24. Min JH, Waters P, Vincent A, Lee S, Y Shin H, H Lee K and Kim BJ: Reduced serum uric acid levels in neuromyelitis optica: Serum uric acid levels are reduced during relapses in NMO. Acta Neurol Scand 126: 287-291, 2012.
25. Lord J, Willis S, Eatock J, Tappenden P, Trapero-Bertran M, Miners A, Crossan C, Westby M, Anagnostou A, Taylor S, et al: Economic modelling of diagnostic and treatment pathways in National Institute for Health and Care Excellence clinical guidelines: The modelling algorithm pathways in guidelines (MAPGuide) project. Health Technol Assess 17: v-vi, 1-192, 2013.

26. Wichtowski M, Nowaczyk P, Kocur J and Murawa D: Irreversible electroporation in the treatment of locally advanced pancreas and liver metastases of colorectal carcinoma. Contemp Oncol (Pozn) 20: 39-44, 2016.

27. Froud T, Venkat SR, Barbery KJ, Gunjan A and Narayanan G: Liver function tests following irreversible electroporation of liver tumors: Experience in 174 procedures. Techn Vasc Interv Radiol 18: 140-146, 2015.

28. Silk MT, Wimmer T, Lee KS, Srimathveeravalli G, Brown KT, Kingham PT, Fong Y, Durack JC, Sofocleous CT and Solomon SB: Percutaneous ablation of peribiliary tumors with irreversible electroporation. J Vasc Interv Radiol 25: 112-118, 2014.

29. Kingham TP, Karkar AM, D'Angelica MI, Allen PJ, Dematteo RP, Getrajdman GI, Sofocleous CT, Solomon SB, Jarnagin WR and Fong Y: Ablation of perivascular hepatic malignant tumors with irreversible electroporation. J Am Coll Surg 215: 379-387, 2012.

30. Thomson KR, Cheung W, Ellis SJ, Federman D, Kavnoudias H, Loader-Oliver D, Roberts S, Evans P, Ball C and Haydon A: Investigation of the safety of irreversible electroporation in humans. J Vasc Interv Radiol 22: 611-621, 2011.

31. Sugimoto K, Moriyasu F, Kobayashi Y, Saito K, Takeuchi H, Ogawa S, Ando M, Sano T, Mori T, Furuichi Y and Nakamura I: Irreversible electroporation for nonthermal tumor ablation in patients with hepatocellular carcinoma: Initial clinical experience in Japan. Jpn J Radiol 33: 424-432, 2015.

32. Cannon R, Ellis S, Hayes D, Narayanan G and Martin RC II: Safety and early efficacy of irreversible electroporation for hepatic tumors in proximity to vital structures. J Surg Oncol 107: 544-549, 2013

33. Hosein PJ, Echenique A, Loaiza-Bonilla A, Froud T, Barbery K, Rocha Lima CM, Yrizarry JM and Narayanan G: Percutaneous irreversible electroporation for the treatment of colorectal cancer liver metastases with a proposal for a new response evaluation system. J Vasc Interv Radiol 25: 1233-1239.e2, 2014.

34. Distelmaier M, Barabasch A, Heil P, Kraemer NA, Isfort P, Keil S, Kuhl CK and Bruners P: Midterm safety and efficacy of irreversible electroporation of malignant liver tumors located close to major portal or hepatic veins. Radiology 285: 1023-1031, 2017.

35. Zhang Q and Teng G: Interventional therapy of colorectal liver metastasis. Zhonghua Wei Chang Wai Ke Za Zhi 20: 621-624, 2017 (In Chinese)

36. Lyu T, Wang X, Su Z, Shangguan J, Sun C, Figini M, Wang J, Yaghmai V, Larson AC and Zhang Z: Irreversible electroporation in primary and metastatic hepatic malignancies: A review. Medicine (Baltimore) 96: e6386, 2017.

37. Washington $\mathrm{K}: 7$ th edition of the AJCC cancer staging manual: Stomach. Ann Surg Oncol 17: 3077-3079, 2010.

38. Edge SB and Compton CC: The American Joint Committee on Cancer: The 7th edition of the AJCC cancer staging manual and the future of TNM. Ann Surg Oncol 17: 1471-1474, 2010. 